\title{
Ionospheric biases correction for coordinates derived from GPS single point positioning
}

\author{
Mauricio Gende $\left({ }^{1}\right)\left({ }^{4}\right)$, Elsa Mohíno Harris $\left({ }^{2}\right)$, Claudio Brunini $\left({ }^{1}\right)$, Sandro M. Radicella $\left({ }^{3}\right)$ \\ and Miguel Herraiz $\left(^{2}\right)$ \\ (') Facultad de Ciencias Astronómicas y Geofísicas, Universidad Nacional de La Plata, Argentina \\ ${ }^{(2)}$ Departamento de Geofísica y Meteorología, Universidad Complutense de Madrid, Spain \\ $\left(^{3}\right)$ Aeronomy and Radiopropagation Laboratory, \\ The Abdus Salam International Centre for Theoretical Physics (ICTP), Trieste, Italy \\ $\left({ }^{4}\right)$ Consejo Nacional de Investigaciones Científicas y Técnicas, Argentina
}

\begin{abstract}
Most GPS users employ low cost receivers. These receivers do not allow users to record the pseudorange that they observe, but the computed coordinates. This work presents an original and simple method to correct ionospheric biases introduced in GPS signals. The originality of this method is based on the fact that no pseudorange is needed to correct the biases, only the calculated coordinates are used. This distinguishes this method from other classic alternatives. This paper evaluates the efficiency of the method with the use of real data.
\end{abstract}

Key words GNSS - Global Positioning System - single point positioning - ionospheric biases correction

\section{Introduction}

The Global Positioning System (GPS) is the most developed and extended Global Navigation Satellite System (GNSS). Its field of application ranges from sub-centimetre level accurate geodynamic studies to decametre level stand-alone positioning. Stand-alone positioning, perhaps the most extended use of GPS, is normally performed using inexpensive receivers that do not record the observed GPS signal measurements, but the coordinates.

Mailing address: Dr. Elsa Mohíno Harris, Departamento de Geofísica y Meteorología, Universidad Complutense de Madrid, Avenida Complutense s/n, 28040 Madrid, Spain; e-mail: emohino@fis.ucm.es
It is well known that the accuracy of GPS positioning is degraded by several biases (Seeber, 2003). Some of these biases are: delays caused by the atmosphere (ionosphere and troposphere), satellite clock and orbit inaccuracy and spurious signal reflection (multi-path). The main bias is the ionospheric delay, which is typically one order of magnitude greater than any of the other afore mentioned biases (Mannucci et al., 1999).

In order to mitigate the ionospheric bias effect on positioning, GPS receivers use an embedded ionospheric model called Ionospheric Correction Algorithm (ICA) (Klobuchar, 1987). The GPS user community has developed several alternative methods to correct this effect. Generally, these methods work in post-processing mode. Therefore, the recording of the observed satellite-to-receiver pseudoranges is required. Other methods designed for real time positioning require at least a real time GPS correction provider and a communication link between the user and the provider. 
This paper will present a post-processing method to mitigate the effect of the ionospheric bias on positioning that does not require the observed pseudorange but only the user coordinates and the Pseudo Random Number (PRN) of the observed satellites. The method does not depend on the ionospheric model used and it accepts corrections from any algorithm that models the ionosphere.

\section{Low precision positioning techniques}

A detailed review of the GPS characteristics, positioning algorithms and different biases affecting the observations can be found in several classical texts (e.g., Hofmann-Wellenhof et al., 2001). Three of the most common single-epoch positioning techniques will be briefly summarized in the following sections.

\subsection{Stand-alone positioning}

This is the simplest positioning method in which coordinates are estimated based on range measurements derived from satellite to receiver travel time. Satellite positions, i.e. the ephemeris, are known since they are encoded in the broadcasted information. Theoretically, having three simultaneous range measurements the problem can be solved by trilateration. However synchronization error due to the receiver clock adds one unknown and consequently at least one extra measurement is needed. In addition to the ephemeris information, the system broadcasts predicted ionospheric parameters in order to reduce the ionospheric error using the ICA model.

\subsection{Precise post-processing point positioning}

This technique differs form the previous one only by the fact that it includes post processed information. Precise ephemeris and satellite clock corrections replace the broadcast ones. An ionospheric delay estimation based on continuously updated global or regional ionospheric models replaces the ICA model. As a result of including this improved information co-
Table I. Standard deviation error for the different positioning techniques.

\begin{tabular}{lcc}
\hline \hline & $\begin{array}{c}\text { Horizontal } \\
\text { error }(\mathrm{m})\end{array}$ & $\begin{array}{c}\text { Vertical } \\
\text { error (m) }\end{array}$ \\
\hline Point positioning & 3.5 & 12.5 \\
Precise point positioning & 2 & 3 \\
Differential positioning & 0.2 & 0.75 \\
\hline
\end{tabular}

ordinates are more accurately determined (Héroux and Kouba, 1995). The main drawback of this technique is the need to record the observed pseudorange, which is not possible with many inexpensive receivers.

\subsection{Differential GPS positioning (DGPS)}

The basic idea behind differential positioning (Blackwell, 1986) is to correct the observed pseudoranges in one GPS receiver (rover station) provided that these corrections could be deduced from another receiver placed in a fixed point with well known coordinates (reference station). DGPS can work either in post-processing or real time mode. In the first case, two receivers observing and recording pseudorange measurements are needed simultaneously. In the second case, a correction provider and a communication link between the user and the provider are required.

\subsection{Comparison between the three techniques}

Table I shows typical values for horizontal and vertical errors that result from point positioning, precise point positioning and differential positioning. Although these particular values were acquired during only a $24 \mathrm{~h}$ period with a sample rate of $30 \mathrm{~s}$, they represent typical errors for each technique.

\section{Algorithm to mitigate the effect of the ionos- pheric bias on positioning}

The idea of the proposed correction algorithm can be summarized as follows: coordi- 
nates could be corrected from ionospheric error by a geometric propagation of an ionospheric biases estimation. Note that in this process no observed range is needed.

\subsection{Error propagation into coordinates}

The basic equation of observation for each range measurement is

$$
\hat{\rho}=\rho-c\left(\delta t^{s}-\delta t_{R}\right)-v
$$

$\hat{\rho}$ being the satellite-to-receiver observed pseudorange, $\rho$ the true geometric range, $c$ the speed of light in vacuum, $\delta t^{S}$ and $\delta t_{R}$ the synchronization errors of the satellite and receiver clocks respectively, $v$ is the total budget of remaining error that biases the measurements.

If $\rho=\rho_{0}+d \rho$ and $d \rho \cong-\cos \alpha \delta n_{R}+$ $-\cos \beta \delta e_{R}-\cos \gamma \delta v_{R}$, where $\cos \alpha, \cos \beta, \cos \gamma$ are the director a cosine, (3.1) transforms into

$\hat{\rho}-\rho_{0}+c \delta t^{s}+v=-\cos \alpha \delta n_{R}-\cos \beta \delta e_{R}+$ $-\cos \gamma \delta v_{R}+c \delta t_{R}$

If there are simultaneous observations to $n$ satellites, using matrix notation (3.2) transforms into

$$
\Delta \rho=A \Delta x
$$

with $A=\left[\begin{array}{cccc}-\cos \alpha^{1} & -\cos \beta^{1} & -\cos \gamma^{1} & c \\ \ldots & \ldots & \ldots & \ldots \\ \ldots & \ldots & \ldots & \ldots \\ \ldots & \ldots & \ldots & \ldots \\ -\cos \alpha^{n} & -\cos \beta^{n} & -\cos \gamma^{n} & c\end{array}\right]$

and $\Delta \boldsymbol{x}=\left(\delta n_{R}, \delta e_{R}, \delta v_{R}, \delta t_{R}\right)^{T}$

This linear system can be solved by least squares

$$
\Delta \boldsymbol{x}=\left(A^{T} A\right)^{-1} A^{T} \Delta \rho .
$$

Finally, the position vector $\hat{\boldsymbol{x}}$, can be found by iteration using $\hat{\boldsymbol{x}}=\boldsymbol{x}_{0}+\Delta \boldsymbol{x}$. Where $\boldsymbol{x}_{0}$ has a first guess value or is to the value obtained in the previous iteration step.

It is important to note that eq. (3.4) represents the way that errors are propagated in the coordinate. If $\hat{\rho}=\rho_{0}=\delta t^{s}=0, v=v_{\text {ionosphere }}$ and $\Delta \boldsymbol{\rho}=\Delta \boldsymbol{\nu}_{\text {ionosphere }}$ then the error on the coordinates due to the ionosphere can be easily computed using eq. (3.4)

$$
\Delta \boldsymbol{x}_{\text {ionosphere }}=\left(A^{T} A\right)^{-1} A^{T} \Delta \boldsymbol{\nu}_{\text {ionosphere }} .
$$

\subsection{Method to correct ionospheric biases at coordinate level}

Ionospheric bias is proportional to Slant Total Electron Content (STEC). This relation can be expressed as: $\Delta \boldsymbol{\nu}_{\text {ionosphere }}=K_{1} \times \mathrm{STEC}$, where $K_{1}$ is a constant and STEC is defined as the integral of free electrons density along the satellite-receiver ray path. There are several ways to estimate STEC values, the present work used Global Ionospheric Maps (GIMs) produced by the Center for Orbit Determination in Europe (CODE) (Schaer et al., 1996). GIMs provide worldwide grids of Vertical Total Electron Content (VTEC); they are computed using a global network of dual-frequency GPS receivers. CODE GIMs are available at $<$ http://www.cx.unibe.ch/aiub/ionosphere.html>. In order to convert VTEC values into STEC values an appropriate mapping function was used: $M=\mathrm{STEC} / \mathrm{VTEC} \cong 1 / \sqrt{1-\cos ^{2}\left(K_{2} E\right)}$ where $K_{2}$ is a constant and $E$ is the satellite elevation.

Having access to STEC estimations from GIMs an ionospheric correction for each pseudorange can be computed as

$$
\Delta \tilde{\boldsymbol{\nu}}_{\text {ionosphere }}=M \times \mathrm{VTEC}^{\mathrm{CODE}-\mathrm{GIMs}} .
$$

Using eq. (3.5) the impact of those STEC estimations on the coordinates is obtained

$$
\Delta \tilde{\boldsymbol{x}}_{\text {ionosphere }}=\left(A^{T} A\right)^{-1} A^{T} \Delta \tilde{\boldsymbol{\nu}}_{\text {ionosphere }} .
$$

Users with a low-cost GPS receiver will not have access to each pseudorange measurement, but only to the final biased coordinates $\hat{\boldsymbol{x}}$ which can be written as $\hat{\boldsymbol{x}}=\boldsymbol{x}+\Delta \boldsymbol{x}_{\text {ionosphere }}+$ $+\Delta \boldsymbol{x}_{\text {other_biases }}$ where $\boldsymbol{x}$ is the exact or true value, evidently not accessible, and usually $\Delta \boldsymbol{x}_{\text {ionosphere }} \gg$ $\gg \Delta \boldsymbol{x}_{\text {other biases }}$.

Finally, if $\Delta \tilde{\boldsymbol{x}}_{\text {ionosphere }}$ is provided it can be subtracted from $\hat{\boldsymbol{x}}$ and a more accurate position can be found 
$\hat{\boldsymbol{x}}_{\text {improved }}=\hat{\boldsymbol{x}}-\Delta \tilde{\boldsymbol{x}}_{\text {ionosphere }}=\boldsymbol{x}+\Delta \boldsymbol{x}_{\text {ionosphere }}+$

$-\Delta \tilde{\boldsymbol{x}}_{\text {ionosphere }}+\Delta \boldsymbol{x}_{\text {other_biases }}$.

Compared to the methods presented in Sections 2.2 and 2.3, the one proposed in this paper does not require access to the pseudorange. This is a clear advantage for situations where pseudoranges are not available, for instance when inexpensive Original Equipment Manufacturer (OEM) GPS boards are used for vehicle tracking.

The algorithm presented in this paper takes it for granted that the satellite geometry contained in matrix $\boldsymbol{A}$ is the same for the user biased coordinates $\hat{\boldsymbol{x}}$, and for the estimated ionospheric correction, $\Delta \hat{\boldsymbol{x}}_{\text {ionosphere }}$. If this assumption is wrong the correction will lose precision; thus, caution must be taken to ensure that the satellites involved in the positioning algorithm are the same as those involved in the correction procedure.

\section{Results}

\subsection{Data set}

The results shown in this section are based on nearly 360000 samples. Two GPS stations were used, located one at geomagnetic mid latitude, California, and the other one at geomagnetic low latitude, Galapagos Islands. Two time periods were analysed, the solstice and the equinox. For each period a set of thirty days with non perturbed ionospheric conditions was selected. All data correspond to a high solar activity period, the year 2001. To avoid problems due to poor signal-to-noise ratio, an elevation mask of $10^{\circ}$ was imposed. Epochs with poor satellite geometry distribution (dilution of precision greater than 6) were not processed.

In order to evaluate the performance of the algorithm four positioning strategies were used: 1) stand-alone positioning without any correction (raw); 2) stand-alone positioning applying ICA correction (ICA); 3) stand-alone positioning using the ionospheric free linear combination (ion free); 4) coordinates correction using a ionospheric model (CC).

The first approach represents a situation where the user has no access to any ionospheric correction. The second one corresponds to the user that applies the ionospheric correction provided by the GPS system. This should be the standard way of point positioning, but unfortunately not all GPS receivers apply the ICA corrections. In the third case the user has a double frequency receiver and can eliminate almost all the ionospheric bias (Hartmann and Leitinger, 1984). Note that this latter case is extremely unusual since double frequency receivers are very expensive and usually are not used in point positioning. This situation was included only to show the magnitude of the error that still remains even when more that $99 \%$ of the ionospheric effect was eliminated. The last approach uses the method introduced in this paper. In this case GIMs maps are used in order to estimate VTEC.

In order to mitigate other sources of biases both precise ephemerides and a tropospheric model were used in the four presented techniques.

\subsection{Algorithm performance}

Table IIa,b presents the accuracy of each positioning method for Galapagos and California respectively. Results agree with some well known facts about the ionosphere. Raw errors clearly show that the ionosphere is more active during the equinox and at low latitudes. ICA and CODE errors demonstrate that, because of its complexity, it is more difficult to estimate the ionosphere during the equinox and at low latitudes. Results also agree with the positioning theory. Ion free errors are independent of the epoch but depend on the noise of the $P$ code, which is clearly higher in the Galapagos receiver. As expected, due to the geometric dilution factor the vertical component is the one that tends to absorb the main part of the ionosphere.

It is worth noting that the method works well, in fact, though the aim of the present work does not aim at comparing or assessing the quality of any particular ionospheric model, it provided a better result that the ICA corrections. CC columns show similar results compared to ion free ones for the vertical, with the exception of the equinox period in Galapagos, probably due to the complexity of the VTEC for that region in that period. But larger dis- 
Table IIa,b. Coordinate errors in meters for each positioning technique at Galapagos Islands (a) and at California (b).

\begin{tabular}{|c|c|c|c|c|c|c|c|c|c|}
\hline & & & & ice & & & Equ & hox & \\
\hline & & Raw & ICA & $\mathrm{CC}$ & Ion free & Raw & ICA & $\mathrm{CC}$ & Ion free \\
\hline (a) & North & 1.72 & 1.58 & 1.30 & 0.68 & 3.41 & 3.56 & 2.62 & 0.70 \\
\hline & East & 1.45 & 1.24 & 1.29 & 0.88 & 2.12 & 1.78 & 1.84 & 0.98 \\
\hline & Vertical & 10.26 & 3.89 & 2.56 & 2.16 & 15.52 & 4.24 & 4.58 & 2.21 \\
\hline (b) & North & 2.32 & 2.04 & 0.66 & 0.70 & 2.50 & 1.71 & 1.21 & 0.67 \\
\hline & East & 1.01 & 0.80 & 0.54 & 0.52 & 1.18 & 0.82 & 0.67 & 0.52 \\
\hline & Vertical & 7.78 & 2.12 & 1.30 & 1.42 & 11.90 & 2.89 & 1.92 & 1.26 \\
\hline
\end{tabular}
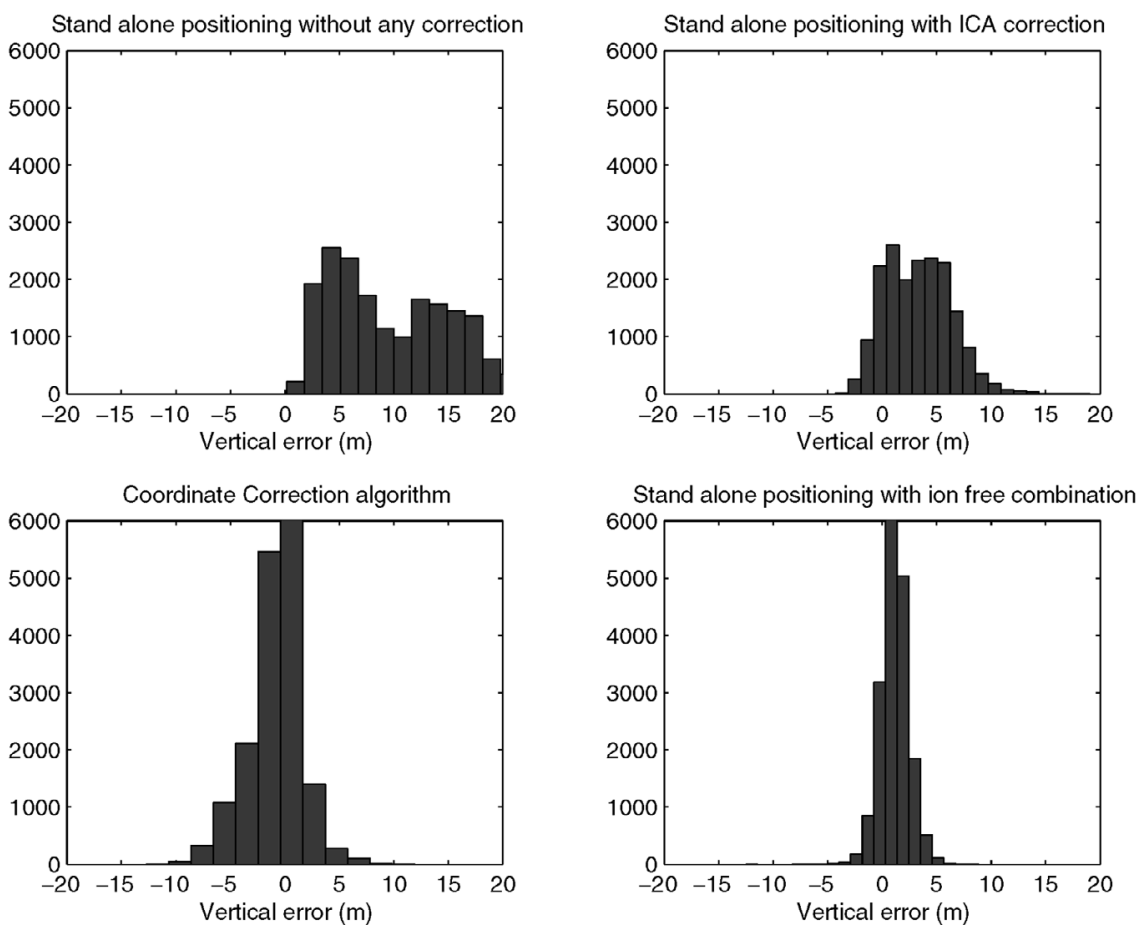

Fig. 1. Histograms of vertical errors for Galapagos during the solstice.

crepancy values arise for the horizontal components. This is probably due to the fact that in order to model the horizontal components correctly, good horizontal gradients of VTEC must be provided (Gende et al., 2003).
CC method was also compared against post process precise point positioning. Differences are in the order of $10 \mathrm{~cm}$ for the horizontal component and in the order of $40 \mathrm{~cm}$ for the vertical component. Figure 1 shows four his- 
tograms of vertical errors for Galapagos station. Data correspond to the first week of July 2001 and around 18000 samples are plotted. Each graph illustrates epoch by epoch residuals for raw, ICA, CC and ion free technique. The first plot clearly illustrates the fact that the ionosphere increases the height of the GPS station. The second one shows that the ICA model partially corrects this effect. The third plot corresponds to the positioning approach presented in this paper. Noticeably better than the first two approaches, it slightly tends to overcorrect the ionospheric effect. The last plot presents a better shape but has some outlier values; these are probably due to the $P$ code signal combination necessary to make ion free solution.

\section{Conclusions}

Results show that the proposed methodology is capable of improving GPS positioning accuracy even when no pseudoranges are recorded. The method presented in this work improves the accuracy of positioning with low cost GPS equipment without any extra expense. Improvements have the same magnitude as those ones obtained in precise point positioning; approximately $7 \mathrm{~m}$ for the horizontal components and $11 \mathrm{~m}$ for the vertical. This represents an improvement of $80 \%$ if no correction is applied or $40 \%$ if ICA correction is applied. Although the proposed methodology cannot present results as good as DGPS, it can be an alternative for users who, needing higher accuracy do not have access to a DGPS correction provider and cannot record pseudoranges for precise point positioning. This can be the case for many low-priced Original Equip- ment Manufacturer (OEM) GPS boards that are typically used in vehicle tracking.

\section{Acknowledgements}

Data from the IGS (International GPS Service) permanent network of GPS receivers were used in this work. These data were obtained thanks to the University of San Diego FTP server (ftp://lox.ucsd.edu/pub/rinex).

\section{REFERENCES}

BLACKWELL, E. (1986): Overview of differential GPS methods, Navigation Red Book, vol. III, 89-100.

Gende, M., S.M. Radicella, B. Nava and C. Brunini (2003): Ionospheric effect in instantaneous positioning, in Institute of Navigation Technical Meeting, January 22-24, Anaheim, California.

Hartmann, G.K. and R. Leitinger (1984): Range errors due to ionospheric and tropospheric effects for signal frequencies above $100 \mathrm{MHz}$, Bull. Geod., 58, 109-136.

HÉrouX, P. and J. KoubA (1995): GPS precise point positioning with a difference, presented at Geomatics '95, June 13-15, 1995, Ottawa, Ontario, Canada.

Hofmann-Wellenhof, B., H. LichtenegGer and J. ColLINS (2001): Global Positioning System: Theory and Practice (Springer Verlag), 5th revised edition, pp. 382.

KLOBUCHAR, J.A. (1987): Ionospheric time-delay algorithm for single-frequency GPS users, IEEE Trans. Aerosp. Electron. Syst., AES-23 (3), 325-331.

Mannucci, A.J., B.A. IIJima, U.J. LindQwister, X. Pi, L. SPARKS and B.D. WILSON (1999): GPS and ionosphere, in Reviews of Radio Science 1996-1999, edited by W.R. STONE (URSI), 625-665.

Schaer S., G. BEUTLER, M. Rothacher and T.A. SPringer. (1996): Daily global ionosphere maps based on GPS carrier phase data routinely produced by the CODE analysis enter, in IGS Analysis Center Workshop, NOAA, Silver Spring, U.S.A.

SeEber, G. (2003): Satellite Geodesy (Walter de Gruyter), 2nd edition, pp. 589 